\title{
Lesestrategien zur Unterstützung des Verstehens von Textaufgaben. Vermittlung und Routinen im Mathematikunterricht aus Sicht von Lehrkräften und Lernenden
}

\author{
Anke Schmitz (iD Fabiana Karstens
}

Eingegangen: 1. Oktober 2020 / Angenommen: 20. Juli 2021 / Online publiziert: 17. August 2021

(C) Der/die Autor(en) 2021

Zusammenfassung Lesestrategien sind bedeutsam, wenn Textaufgaben zu bearbeiten sind und die in der Aufgabe dargestellte Situation verstanden werden muss. Die Studie betrachtet die Vermittlung von Lesestrategien im Mathematikunterricht aus Sicht von Lehrkräften sowie Lernenden und untersucht, in welcher Relation die Sichtweisen stehen. Im Fokus stehen beide Perspektiven, um basierend auf vorliegenden Konvergenzen Aufschluss über Unterrichtsroutinen zu erhalten und angesichts zunehmender Heterogenität differenzielle Wahrnehmungen in der Gruppe der Lernenden zu ermitteln. 60 Lehrpersonen und 261 Lernende wurden zur Vermittlung von (meta-)kognitiven Lesestrategiearten, der Instruktionsweise bei der Strategievermittlung sowie zur Relevanz von Lesestrategien im Unterricht befragt. Während zwischen beiden Perspektiven Konvergenzen in der Wahrnehmung der Vermittlung kognitiver Lesestrategien sowie der Relevanz der Thematik bestehen, unterscheiden sie sich bezüglich der Vermittlung metakognitiver Lesestrategien sowie der Instruktionsweise. Zudem zeigt eine profilspezifische Betrachtung der Wahrnehmungen innerhalb der Lernenden, dass sie die Vermittlung von Lesestrategien im Mathematikunterricht in Abhängigkeit des Leseverständnisses differenziell einschätzen: Lernende mit schlechteren Leseverständniswerten nehmen die Vermittlung von Lesestrategien im Unterricht in höherem Maße wahr als die Lernenden mit besseren Leseverständniswerten.

\footnotetext{
Anke Schmitz $(\bowtie)$

Institut für Deutsche Sprache und Literatur und ihre Didaktik, Leuphana Universität Lüneburg, Universitätsallee 1, 21335 Lüneburg, Deutschland

E-Mail: anke.schmitz@leuphana.de

Fabiana Karstens

Institut für deutsche Sprache und Literatur II, Universität zu Köln,

Albertus-Magnus-Platz, 50923 Köln, Deutschland
} 
Schlüsselwörter Lesestrategien · Unterrichtsforschung · Mathematik ·

Textaufgaben

\title{
Reading Strategies for the Comprehension of Text-based Tasks. Instructional Practices and Routines in Mathematics from Teachers' and Students' Perspectives
}

\begin{abstract}
Reading strategies are important for the solution of text-based tasks and the comprehension of the situation presented in the task. The study investigates to which extent reading strategies are part of classroom instruction by asking how teachers and students perceive the strategy instruction. Both perspectives are included to get insight into overlaps as indicators for actual classroom action and in terms of increasing heterogeneity to discover differential perceptions within students. Therefore, 60 teachers and 261 students were asked about their perceptions of strategy instruction. Besides cognitive and metacognitive reading strategies, and type of strategy instruction, they were asked about the relevance of this topic in class. While the perspectives overlap in terms of cognitive reading strategies and the relevance of the topic in general, they differ regarding metacognitive reading strategies and the type of strategy instruction. Furthermore, a profile-specific approach of students' perspectives shows differential perceptions of reading strategy instruction in mathematics lessons depending on their level of reading comprehension: Students with low reading competence approve a larger amount of reading strategy instruction than students with higher reading competence.
\end{abstract}

Keywords Reading strategies $\cdot$ Classroom research $\cdot$ Mathematics $\cdot$ Text-based tasks

\section{Einleitung}

Die Ergebnisse der PISA-Studie 2018 verdeutlichen, dass sich der Anteil an Lernenden, welcher die mathematische Kompetenzstufe II nicht erreicht, vergrößert hat (Reinhold et al. 2019). Dies betrifft vor allem Lernende an nicht-gymnasialen Schularten, eine Gruppe, die zugleich über eingeschränkte Lesefähigkeiten verfügt (Weis et al. 2019) und mit erschwerten Bedingungen in Schule, Ausbildung und Beruf zu rechnen hat (Reinhold et al. 2019). Für den Mathematikunterricht sind unzureichende Lesekompetenzen nachteilig, da die Bedeutung von sprachlichen Lernvoraussetzungen für mathematische Leistungen wiederholt nachgewiesen wurde (Heinze et al. 2011; Prediger et al. 2015). Insbesondere die Bearbeitung von Textaufgaben beruht auf einem adäquaten Verstehen des mathematischen Textes (Blum und Leiss 2005; Reusser 1989), was viele Lernende vor Herausforderungen stellt (u.a. Dröse und Prediger 2019; Plath 2019; Schlager 2020).

Einen Ansatzpunkt zur Förderung des Verstehens von Textaufgaben bietet die Vermittlung von Lesestrategien (Dignath und Büttner 2018; Schlager 2020; Schukajlow und Leiss 2011). Ob und welche Lesestrategien üblicherweise Gegenstand von Mathematikstunden sind, in denen Textaufgaben bearbeitet werden, wurde noch 
nicht untersucht. Die Forschung konzentrierte sich bislang auf das selbstregulierte Lernen (Berger und Karabenick 2011; Kistner et al. 2015; Leiss et al. 2007) oder betrachtete metakognitive Aktivtäten und diskursive Prozesse im mathematischen Lösungsprozess (Cohors-Fresenborg und Kaune 2003) mithilfe von punktuellen Beobachtungen (Dignath und Büttner 2018; Kistner et al. 2010, 2015).

Die vorliegende Studie stellt insofern eine Erweiterung dar, indem beleuchtet wird, welche Lesestrategien im Mathematikunterricht an weiterführenden Schulen vermittelt werden, wenn Textaufgaben im Mittelpunkt der Stunde stehen und welche unterrichtlichen Routinen vorliegen (Erath et al. 2021). Um einen Einblick in das Unterrichtshandeln zu erhalten, wurden Lehrkräfte und Lernende zu ihren Langzeiterfahrungen befragt und ihre Antworten hinsichtlich Konvergenzen und Differenzen verglichen (Baumert et al. 2004; Maßß und Engeln 2018). Vor allem Übereinstimmungen in der Wahrnehmung können als Indikatoren dafür gelten, in welchem Maß das von den Lehrkräften subjektiv gestaltete Angebot tatsächlich im Unterrichtsalltag präsent ist (Baumert et al. 2004; Maaß und Engeln 2018). Ausgehend von Befunden, dass Unterricht von Lernenden angesichts ihrer heterogenen Lernvoraussetzungen differenziell wahrgenommen wird (Holzberger et al. 2016) und ihre Wahrnehmung einen bedeutsamen Beitrag zur Vorhersage von Lernoutcomes leistet, wird zudem betrachtet, wie Lernende die Strategievermittlung in Abhängigkeit ihrer Lernvoraussetzungen wahrnehmen.

Im Folgenden wird zunächst auf das Verstehen von Textaufgaben und die Funktionen sowie Arten von Lesestrategien eingegangen (Abschnitt 2). Nach der Beschreibung unterrichtlicher Vermittlungsprinzipien und der Forschung zum Unterrichtshandeln in Mathematik bezogen auf selbstregulative Kompetenzen (Abschnitt 3) werden die Fragestellungen (Abschnitt 4), das methodische Vorgehen der Fragebogenstudie sowie die Schritte der Datenauswertung präsentiert (Abschnitt 5). Nach sukzessiver Darstellung der Befunde (Abschnitt 6) werden die Ergebnisse in den Forschungsstand eingeordnet und Limitationen der Studie aufgezeigt.

\section{Textaufgaben und die Bedeutung von Lesestrategien in Mathematik}

\subsection{Der Verstehensprozess von Textaufgaben und seine Herausforderungen}

Mathematische Textaufgaben sind eine Aufgabenart des Sachrechnens, bei der mathematische Sachverhalte in sprachliche Darstellungen eingebettet sind. Beim Sachrechnen werden verschiedene Aufgabentypen differenziert, wie bspw. eingekleidete Aufgaben, Textaufgaben und Modellierungs-/Sachaufgaben (Plath 2019; Schlager 2020).

In diesem Beitrag geht es insbesondere um das Verstehen von Problemsituationen auf der Grundlage von Textaufgaben, das durch ein Zusammenspiel aus Alltagsverständnis, Situationsverständnis und Rechenfertigkeiten gekennzeichnet ist (Blum und Leiss 2005; Reusser 1989). Gemäß der kognitionspsychologischen Leseforschung ist davon auszugehen, dass Lernende nicht nur bei Modellierungsaufgaben, sondern auch beim Verstehen von Textaufgaben ein Situationsmodell (Kintsch und Greeno 1985; Kintsch 1998) bilden, womit die im Aufgabentext dargestellte Situa- 
tion vollumfänglich nachvollzogen wird (Leiss et al. 2010). Ein Situationsmodell umfasst eine vom Wortlaut des Textes unabhängige Repräsentation des Textinhaltes, wofür Leserinnen und Leser verstärkt Vorwissen sowie Erfahrungen integrieren und Schlussfolgerungen (Inferenzen) ziehen müssen. Dabei finden interaktive bottom-up und top-down-Prozesse statt (Kintsch 1998; Richter und Schnotz 2018). Bottom-up Verstehensprozesse werden durch semantische, syntaktische und strukturelle Texteigenschaften beeinflusst (Reusser 1989). Zugleich erfolgen top-down Verstehensprozesse, die durch kognitive und motivationale Voraussetzungen der Lernenden bestimmt werden, darunter ihre Lesekompetenz, ihr inhaltliches Vorwissen und Lesestrategiewissen sowie ihre allgemeinen mathematischen Grundfähigkeiten (Leiss et al. 2019). Bevor jedoch ein adäquates Situationsmodell konstruiert werden kann, ist auf der Grundlage einer Oberflächenrepräsentation eine propositionale Repräsentation zu erzeugen (Kintsch 1998). Dabei wird der semantische Gehalt des Textes durch Kohärenzbildungsprozesse von der Satzebene hin zur Textebene komprimiert und unter Einbezug von Vorwissen in ein Situationsmodell überführt. Dieses wird je nach Aufgabenanforderungen vereinfacht bzw. strukturiert und in ein mathematisches Modell überführt, indem ggf. überflüssige Informationen im Aufgabentext identifiziert oder fehlende Informationen durch eigene Angaben ergänzt werden. Solche Angaben können allerdings nur dann korrekt identifiziert werden, wenn die Lernenden in ihrem Situationsmodell Inkongruenzen wahrnehmen (Böckmann und Schukajlow 2020). Fokussieren Lernende hauptsächlich auf die im Text erwähnten Zahlen und/oder (vermeintlichen) Schlüsselwörter, und nehmen sie voreilig Interpretationen vor (Dröse 2020; Dröse und Prediger 2019; Prediger und Krägeloh 2015; Verschaffel et al. 2000), können sie weder eine kohärente propositionale Repräsentation noch ein adäquates Situationsmodell konstruieren. Die Komplexität des Verstehensprozesses spiegelt sich auch darin wider, dass er bis zu 50\% der Gesamtbearbeitungsdauer einer Textaufgabe einnehmen kann und es bei unzureichendem Verstehen zu enormen Schwierigkeiten in der Aufgabenbearbeitung kommen kann (Leiss et al. 2019; Plath 2019). Im Idealfall sind basierend auf dem Situationsmodell nur noch jene Angaben im mathematischen Modell enthalten, die für die erforderlichen Rechenoperationen erforderlich sind. Liegt abschließend ein Resultat vor, wird dieses hinsichtlich des zuvor konstruierten Situationsmodells inhaltlich validiert. Dafür bedarf es der Reaktivierung des zu Beginn des Bearbeitungsprozesses konstruierten Situationsmodells. Die Konstruktion eines adäquaten Situationsmodells ist somit eine entscheidende Stellgröße für den Lösungsprozess und die korrekte Aufgabenlösung (Böckmann und Schukajlow 2020; Dröse und Prediger 2019; Krug und Schukajlow 2012; Leiss et al. 2010).

\subsection{Funktionen und Arten von Lesestrategien}

Um den Umgang mit Textaufgaben zu unterstützen, sollten Mathematiklehrkräfte die sprachlichen Dimensionen in ihrem Unterricht in den Blick nehmen (Leiss und Plath 2020). Dazu gehört auch, ,,mathematisches Lesen“ (Böckmann und Schukajlow 2020, S. 4) als wichtigen Teilschritt für das Bearbeiten von Textaufgaben zu beachten. Mathematisches Lesen ist gekennzeichnet durch ein genaues Lesen des 
Aufgabentextes sowie die Fokussierung auf Textstrukturen und Zusammenhänge unter Einbezug von Vorwissen (Shanahan et al. 2011).

Aus der kognitionspsychologischen Leseforschung ist bekannt, dass Lesestrategien vor allem hierarchiehöhere Verstehensprozesse, wie den Aufbau einer propositionalen Repräsentation und eines Situationsmodells, begünstigen (Gold 2018; Philipp und Schilcher 2012; Van Dijk und Kintsch 1983). Der Stellenwert von Lesestrategien für die Bearbeitung von Textaufgaben wird auch von der Mathematikdidaktik betont (Bescherer und Papadopoulou 2017; Dröse und Prediger 2019; Hagena et al. 2017; Leiss et al. 2019; Lingel et al. 2014; Plath 2019) - sie werden dort auch als Verstehensstrategien bezeichnet. Unterschieden werden in Anlehnung an Weinstein und Mayer (1986) kognitive, metakognitive und ressourcenbezogene Lesestrategien, von denen insbesondere die beiden erstgenannten Strategiearten relevant sind, da sie sich auf die kognitive Informationsverarbeitung und metakognitive Steuerung des Verstehens richten (Schlager 2020; Schukajlow und Leiss 2011):

- Kognitive Lesestrategien dienen der unmittelbaren Informationsverarbeitung. Unterschieden werden Organisationsstrategien zur Strukturierung vorliegender Informationen (z.B. das Zusammenfassen, das Unterstreichen, das Anfertigen von Visualisierungen und Skizzen, das Notieren bzw. Herausschreiben von Informationen), Elaborationsstrategien zur Verknüpfung des Gelesenen mit eigenem Vorwissen (z. B. das Fragen stellen, das Aktivieren von Vorwissen, das Formulieren von Hypothesen, bildliches Vorstellen) und Wiederholungsstrategien zur genauen Wahrnehmung von Informationen (z. B. mehrmaliges Lesen der Aufgabe).

- Metakognitive Lesestrategien planen, kontrollieren und regulieren den eigenen Strategieeinsatz und den Verstehensprozess. Hierzu zählen die Planung des erforderlichen Strategieeinsatzes, die Überwachung des Verstehens, die Regulierung des Strategieeinsatzes, der Umgang mit unverständlichen Textstellen und die Reflexion des Verstehens.

Wenngleich die Bedeutung der zuvor aufgeführten Lesestrategiearten oder ihr Zusammenwirken hinsichtlich des Lesens von Textaufgaben bislang nicht vollumfänglich untersucht wurde (Schukajlow et al. 2018), wird angenommen, dass kognitive Lesestrategien das Bearbeiten von Textaufgaben unterstützen können. Dazu zählen Organisationsstrategien, die eine bewusste Wahrnehmung der sprachlichen Formulierungen im Aufgabentext (Dröse und Prediger 2019), aber auch die gezielte Identifikation von relevanten Informationen und die Herstellung von Bedeutungen und Zusammenhängen begünstigen (Schlager 2020). Prediger und Krägeloh (2015) untersuchten auch das organisierende Concept-Mapping, welches sich positiv auf die korrekte Rekonstruktion der in der Aufgabe dargestellten Problemsituation bei Lernenden in achten Klassen auswirkte. Zudem weisen Schukajlow und Leiss (2011) darauf hin, dass wiederholtes Lesen, Unterstreichen bzw. Markieren, das Notieren von Angaben sowie das Zeichnen einer Skizze (Letzteres vor allem für die Organisation, Strukturierung und Verknüpfung von Informationen) die Bearbeitung von Textaufgaben stützen. Elaborationsstrategien und metakognitive Lesestrategien, die sich aus Sicht der Textverstehensforschung besonders förderlich auf die Bildung und Überprüfung des Situationsmodells auswirken können (Gold 2018), wurden bislang weniger untersucht, ausgenommen das Treffen von Annahmen (Krug und Schukaj- 
low 2012), das sich positiv auf das Modellieren und das bildliche Vorstellen der Aufgabensituation auswirken kann.

Lesestrategien sind auch in einigen Fördermaterialien integriert (z. B. Bescherer und Papadopoulou 2017) und Studien zeigen, dass Lernende einige Lesestrategien (z.B. Unterstreichen, Notizen machen und Herausschreiben von Angaben, wiederholtes Lesen, bildliches Vorstellen) laut Selbstbericht (Schukajlow und Leiss 2011; Leiss und Plath 2020) bei der Aufgabenbearbeitung auch spontan anwenden. Plath (2019) zeigt allerdings, dass die spontane Strategieanwendung von Lernenden keinen Einfluss auf die Lösungsrate der Aufgabe nimmt. Böckmann und Schukajlow (2020) weisen außerdem nach, dass Lernende aus neunten Klassen Schwierigkeiten mit dem Anfertigen von Notizen und dem Formulieren von Annahmen haben, wohingegen das Markieren relevanter Informationen leichter gelingt. An diesem Punkt rückt die Vermittlung von Lesestrategien und die Frage ins Blickfeld, inwiefern Lehrkräfte im Mathematikunterricht Lesestrategien beim Bearbeiten von Textaufgaben überhaupt vermitteln bzw. welchen Stellenwert die Thematik im Mathematikunterricht einnimmt.

\section{Bedeutung der Vermittlung von Lesestrategien durch Lehrkräfte im Unterricht}

Ungeachtet dessen, ob es um Lern- oder Lesestrategien geht, scheint ein geeigneter Vermittlungszeitpunkt der Beginn der Sekundarstufe I zu sein, da sich strategische Lernweisen in diesem Alter noch gut beeinflussen lassen (Dignath und Büttner 2018; Philipp und Schilcher 2012; Whitebread 2000). Grundsätzlich hat sich eine direkte und explizite Instruktionsweise als zielführend erwiesen, bei der die Lehrkräfte die Anwendung der Strategien modellieren und sie von ihren Lernenden beobachten lassen (Zimmerman 2000) und in ihrem Unterricht auf den Nutzen und die Funktionalität von Strategien hinweisen (Dignath und Büttner 2018; Hamman et al. 2000; Kistner et al. 2010). Weiter wird auf das Konzept des Cognitive Apprenticeship verwiesen, indem neben Phasen direkter Instruktion auch (begleitete) Übungsphasen ermöglicht werden, bis die Lernenden die Strategien eigenständig anwenden können (Duffy 2002; Duke und Pearson 2002; Perry et al. 2008; Zimmerman 2000). Trainingsstudien zur Vermittlung von Lesestrategien weisen außerdem darauf hin, dass kognitive und metakognitive Strategien in Kombination instruiert werden sollten (Schünemann et al. 2013; Souvignier und Mokhlesgerami 2006), da der Einsatz metakognitiver Strategien die Anwendungsqualität kognitiver Lesestrategien erhöhen kann (Leopold und Leutner 2015). Diesen Schluss legt auch die Studie von Hagena et al. (2017) nahe, die mit ihrem überwiegend auf kognitive Lesestrategien ausgerichteten Training keinen Vorsprung in der Trainingsgruppe mit Blick auf den mathematischen Modellierungsprozess erzielten. Bedeutsam ist darüber hinaus, dass Lehrkräfte die instrumentelle Motivierung durch Relevanzsetzung der Thematik adressieren, indem sie ihre Lernenden auf die Bedeutung der Anwendung von Lesestrategien hinweisen (Boekaerts 1999; Dignath und Büttner 2008).

Forschungsarbeiten zum Handeln von Lehrkräften im Mathematikunterricht angesichts der Vermittlung von strategischen Kompetenzen verdeutlichen, dass bislang 
vor allem das selbstregulierte Lernen als domänenübergreifendes Konstrukt untersucht wurde. Dazu wurde überwiegend mit Unterrichtsbeobachtungen, Videografie und Interviewverfahren gearbeitet und insbesondere auf Lehrkräfte fokussiert. Dignath und Büttner (2018) verdeutlichen, dass Mathematiklehrkräfte kognitive Lernstrategien als wichtig erachten, sie überwiegend implizite Instruktionen wählen und kaum metakognitive Lernstrategien im Unterricht anleiten. Kistner et al. (2010) bestätigen dieses Bild durch Re-Analysen eines Subsamples der deutschschweizerischen Videostudie zum Satz des Pythagoras (Klieme et al. 2009). Darüber hinaus konstatieren Kistner et al. (2015) ebenfalls auf Basis der genannten Videostudie, dass Lehrkräfte in Stunden, in denen sie neue Konzepte einführen, tendenziell mehr kognitive Lernstrategien vermitteln und in Übungsstunden, in denen das Lösen von (Text-) Aufgaben im Mittelpunkt steht, mehr metakognitive Lernstrategien instruieren, wenngleich sich die Werte aber auf niedrigem Niveau befinden. Hinsichtlich der Vermittlung von Lernstrategien verweisen die Beobachtungsstudien somit auf Entwicklungspotenzial (Dignath und Büttner 2018), wenngleich es sich jeweils um kleine Stichproben handelt. Studien zur üblichen Vermittlungspraxis von Lesestrategien in Mathematikstunden, in denen Textaufgaben bearbeitet werden, liegen zum aktuellen Zeitpunkt nicht vor.

Um Aufschluss über unterrichtliche Routinen zu erhalten, wird empfohlen, Lehrkräfte und Lernende gleichermaßen zu ihren Langzeiterfahrungen zu befragen (Baumert et al. 2004; Maaß und Engeln 2008), da beide Sichtweisen wertvolle Einblicke in unterrichtliches Handeln ermöglichen und insbesondere die Aufdeckung von Konvergenzen, also die geteilte Wahrnehmung beider Personengruppen, Schlussfolgerungen zulässt, was üblicherweise im Unterricht passiert (Baumert et al. 2004; Maaß und Engeln 2008). Während Lehrkräfte sich für eine methodisch-didaktische, wenngleich subjektiv eingefärbte, Beschreibung ihres unterrichtlichen Angebots und ihrer Absichten eignen, gelten Lernende als Expertinnen und Experten für die valide Beschreibung von unterrichtlichen Lehrroutinen (Clausen 2002; Ditton 2002; Fauth et al. 2014), indem sie reichhaltige Erfahrungen über verschiedene Unterrichtsstunden hinweg aggregieren. Die besondere Relevanz der Lernendenperspektive zeigt sich ferner darin, dass lernendenseitige Wahrnehmungen mit Blick auf unterrichtliche Wirkungszusammenhänge Mediationsprozesse darstellen, die zwischen dem von der Lehrkraft gestalteten Angebot und dem Lernerfolg der Lernenden vermitteln (Helmke 2017). Zudem weist die Unterrichtsforschung darauf hin, dass Unterrichtswahrnehmungen von Lernenden einen bedeutsamen Beitrag zur Vorhersage von Lernerfolgsmerkmalen leisten (Clausen 2002; de Jong und Westerhof 2001; Fauth et al. 2014) und vor allem potenziellen differenziellen Wahrnehmungen besondere Aufmerksamkeit geschenkt werden sollte (Jurik et al. 2015; Seidel 2006).

\section{Fragestellungen}

Die Studie untersucht, welche Lesestrategien Mathematiklehrpersonen beim Bearbeiten von Textaufgaben im Unterricht der fünften Jahrgangsstufe üblicherweise vermitteln. Es geht explizit um solche Unterrichtsstunden, in denen Textaufgaben bearbeitet werden. Um Aufschluss über die unterrichtlichen Routinen zu erhalten, 
wurden Lehrkräfte sowie Lernende zu folgenden Aspekten befragt (vgl. ähnliche Dimensionen in Kistner et al. 2015), die in der vorliegenden Studie explizit auf das Lesen und Verstehen von Textaufgaben ausgerichtet wurden: (1) Vermittlung kognitiver Lesestrategiearten, (2) Vermittlung metakognitiver Lesestrategiearten, (3) Instruktionsweise von Lesestrategien und (4) die instrumentelle Motivierung durch im Unterricht verdeutlichte Relevanz von Lesestrategien für das Bearbeiten von Textaufgaben. Ausgehend hiervon werden folgende Fragestellungen untersucht:

1. Im welchem Maße ist die Vermittlung von Lesestrategien aus der Sicht von Lehrkräften und Lernenden Gegenstand von solchen Stunden des Mathematikunterrichts, in denen Textaufgaben bearbeitet werden, und wo liegen Konvergenzen und Differenzen in der Wahrnehmung des Unterrichts vor?

2. Lassen sich auf Seiten der Lernenden differenzielle Einschätzungen des Unterrichts identifizieren, was kennzeichnet die Lernenden hinsichtlich ihrer kognitiven Lernvoraussetzungen, und in welcher Relation stehen mögliche profilspezifische Einschätzungen zu den Einschätzungen von Lehrkräften?

\section{Methode}

Die Befragungen der Lehrkräfte und Lernenden wurden als ergänzende Erhebungen im Kontext des BiSS-Evaluationsprojekts „Evaluation der Implementation von Leseförderkonzepten in der Sekundarstufe I“ (EILe) durchgeführt. In diesem Projekt wurden 23 weiterführende Schulen drei Jahre lang begleitet und es wurde evaluiert, inwiefern Leseförderkonzepte zur Vermittlung von selbstreguliertem Lesen in den Unterricht implementiert werden. Dazu gehört auch das Fach Mathematik. Teilergebnisse aus der im Beitrag präsentierten Lehrerbefragung sind in Schmitz (2019) veröffentlicht. ${ }^{1}$

\subsection{Stichproben}

Es wurden 261 Lernende aus 21 verschiedenen fünften Klassen in sieben weiterführenden nicht-gymnasialen Schulen befragt. Pro Klasse nahmen durchschnittlich etwa 18 Lernende teil. Sie waren zu 51,5\% weiblich und im Schnitt $M=10,97$ Jahre alt $(S D=0,62)$. Neben ihrem Alter und Geschlecht wurden die Lernenden in einem Fragebogen zu Merkmalen befragt, die in Zusammenhang mit ihrer Lesekompetenz stehen: Als bewährter Indikator für den sozio-ökonomischen Status wurde ein fünfstufiger Buchindex genutzt (Wie viele Bücher habt ihr zu Hause? 1= keine oder nur sehr wenige, 2 = genug, um ein Regalbrett zu füllen, 3= genug, um ein Regal zu füllen, 4 = genug, um drei Regale zu füllen, 5 = über 200 Bücher; vgl. IGLU-2006; Bos et al. 2010; Paulus 2009). Die Muttersprache wurde dichotom kodiert, indem die Lernenden angeben sollten, ob sie Deutsch oder eine andere Sprache als ihre Mutter-

\footnotetext{
1 In Schmitz (2019) wurde die Perspektive von Lehrkräften in drei Fächern (Deutsch, Mathematik, Biologie) verglichen, um zu ermitteln, welche Lesestrategien fachspezifisch oder fachübergreifend in den jeweiligen Fächern unterrichtet werden. Zum Zwecke des Fachvergleichs wurden andere Items für die Berechnungen auf Skalenebene inkludiert.
} 
sprache bezeichnen (IGLU-2006; Bos et al. 2010). Sowohl für das Fach Mathematik als auch für Deutsch wurde die letzte Zeugnisnote erhoben. 74,8\% der Lernenden sprach Deutsch als Muttersprache und sie besaßen $M=3,39(S D=1,18)$ Bücher. Im Fach Mathematik lag die durchschnittliche Note bei $M=2,68(S D=0,89)$, im Fach Deutsch bei $M=2,72(S D=0,68)$. Die Stichprobe lässt sich hinsichtlich dieser Hintergrundmerkmale als durchschnittlich beschreiben.

Bei den Lehrkräften handelte es sich um 60 Mathematiklehrkräfte in den benannten Schulen, die zum Zeitpunkt der Befragung in fünften Klassen unterrichteten bzw. Auskunft über den Unterricht in dieser Schulstufe geben konnten und zu $73,3 \%$ weiblich waren. Ihr Alter betrug $M=41,42$ Jahre $(S D=11,23)$ und sie waren $M=13,89$ Jahre $(S D=10,72)$ im Schuldienst tätig. ${ }^{2}$

\subsection{Erfassung von kognitiven Lernvoraussetzungen der Lernenden}

Um solche kognitiven Lernvoraussetzungen zu erfassen, die sich nachweislich als prädiktiv für das Textverständnis und die Konstruktion eines Situationsmodells erwiesen haben (Cromley und Azevedo 2007) sowie die Wahrnehmung des Unterrichts beeinflussen können (Jurik et al. 2015; Seidel 2006), wurden die kognitive Grundfähigkeit, die Leseflüssigkeit, das Leseverständnis und das Lesestrategiewissen erhoben. Die eingesetzten Verfahren sowie die Testwerte sind in Tab. 1 ersichtlich.

Die Lernenden haben im Wortschatz- und Leseflüssigkeitstest durchschnittliche Werte erzielt. Da bei den anderen beiden Instrumenten aus inhaltlichen und zeitökonomischen Gesichtspunkten Auszüge bzw. Subtests genutzt wurden, sind Einordnungen in Normtabellen nicht möglich. Die Mittelwerte lassen jedoch den Schluss $\mathrm{zu}$, dass die Lernenden durchschnittliche Werte im Leseverständnis aufweisen und im Lesestrategiewissenstest etwas über dem Durchschnitt liegen.

Tab. 1 Erhobene Lernvoraussetzungen der Lernenden

\begin{tabular}{|c|c|c|c|c|}
\hline Lernvoraussetzung & Verfahren & $M(S D)$ & Itemanzahl & $\begin{array}{l}\text { Theoreti- } \\
\text { scher } \\
\text { Range }\end{array}$ \\
\hline $\begin{array}{l}\text { Kognitive } \\
\text { Grundfähigkeit } \\
\text { (verbal) }\end{array}$ & $\begin{array}{l}\text { Wortschatztest des CFT-20R (Weiß } \\
\text { 2006) }\end{array}$ & $\begin{array}{l}16,14 \\
(4,82)\end{array}$ & 30 & $\begin{array}{l}\text { Summen- } \\
\text { score: } \\
0-30\end{array}$ \\
\hline Leseflüssigkeit & $\begin{array}{l}\text { Salzburger Lesescreening (SLS 2-9; } \\
\text { Wimmer und Mayringer 2014) }\end{array}$ & $\begin{array}{l}43,42 \\
(9,73)\end{array}$ & 18 & $\begin{array}{l}\text { Summen- } \\
\text { score: } \\
0-100\end{array}$ \\
\hline Leseverständnis & $\begin{array}{l}\text { Subskala Sachtext des Frankfurter } \\
\text { Leseverständnistests (FLVT 5-6; } \\
\text { Souvignier et al. 2008) }\end{array}$ & $\begin{array}{l}10,36 \\
(3,61)\end{array}$ & 18 & $\begin{array}{l}\text { Summen- } \\
\text { score: } \\
0-18\end{array}$ \\
\hline $\begin{array}{l}\text { Lesestrategie- } \\
\text { wissen }\end{array}$ & $\begin{array}{l}\text { Items aus dem Würzburger } \\
\text { Lesestrategie-Wissenstest (WLST } \\
\text { 7-12; Schlagmüller und Schneider } \\
\text { 2007) }\end{array}$ & $\begin{array}{l}39,31 \\
(9,46)\end{array}$ & $\begin{array}{l}4 \text { Szenarien } \\
\text { à } 5-7 \\
\text { Alternativen }\end{array}$ & $\begin{array}{l}\text { Summen- } \\
\text { score: } \\
0-62\end{array}$ \\
\hline
\end{tabular}

\footnotetext{
2 Da in der Stichprobe der Lehrpersonen aufgrund von Datenschutzbestimmungen in einem Bundesland (vollständige Anonymisierung) keine Klassenidentifikation vorgenommen werden durfte, ist es leider nicht möglich, die Antworten der Lernenden den entsprechenden Lehrkräften zuzuordnen.
} 
Tab. 2 Skalen, Itembeispiele und interne Konsistenz (Cronbachs $\alpha$ ) der Fragebögen

\begin{tabular}{|c|c|c|c|}
\hline $\begin{array}{l}\text { Skala (Item- } \\
\text { anzahl) }\end{array}$ & $\begin{array}{l}\text { Likert- } \\
\text { Skalierung }\end{array}$ & Itembeispiele & $\begin{array}{l}\text { Reliabilität } \\
\text { (Cronbach) L/S }\end{array}$ \\
\hline $\begin{array}{l}\text { Vermittlung } \\
\text { kognitiver } \\
\text { Lesestrate- } \\
\text { giear- } \\
\text { ten (13) }\end{array}$ & Häufigkeit & $\begin{array}{l}\text { L: Wie oft fordern Sie ihre Lernenden beim Lesen } \\
\text { von Textaufgaben im Mathematikunterricht dazu } \\
\text { auf? } \\
\text { S: Wie oft macht ihr im Mathematikunterricht } \\
\text { diese Dinge, wenn ihr Textaufgaben lest? } \\
\text { Visualisieren/Skizzen anfertigen } \\
\text { Notizen machen } \\
\text { Schlüsselwörter, Textstellen, Zahlen markieren } \\
\text { Bildlich vorstellen, worum es geht } \\
\text { Den Inhalt der Aufgabe zusammenfassen }\end{array}$ & $\alpha=0,83 / 0,85$ \\
\hline $\begin{array}{l}\text { Vermittlung } \\
\text { metakogni- } \\
\text { tiver } \\
\text { Lesestrate- } \\
\text { giearten (8) }\end{array}$ & Häufigkeit & $\begin{array}{l}\text { L: Wie oft fordern Sie ihre Lernenden beim Lesen } \\
\text { von Textaufgaben im Mathematikunterricht dazu } \\
\text { auf? } \\
\text { S: Wie oft macht ihr im Mathematikunterricht } \\
\text { diese Dinge, wenn ihr Textaufgaben lest? } \\
\text { Vor dem Lesen einen Plan machen, welche } \\
\text { Lesestrategien sie/wir nutzen können } \\
\text { Während des Lesens überwachen/kontrollieren, ob } \\
\text { sie/wir auch alles verstehen } \\
\text { Nach dem Lesen überlegen, ob sie/wir die richtige } \\
\text { Lesestrategie angewendet haben }\end{array}$ & $\alpha=0,80 / 0,83$ \\
\hline $\begin{array}{l}\text { Instruktions- } \\
\text { qualität bei } \\
\text { der Lese- } \\
\text { strategie- } \\
\text { vermitt- } \\
\text { lung (5) }\end{array}$ & Zustimmung & $\begin{array}{l}\text { L: Bei der Anleitung/Erklärung einer } \\
\text { Lesestrategie gehe ich so vor, dass } \\
\text { Ich die Strategieanwendung erkläre (verbal) } \\
\text { Ich den Nutzen erläutere } \\
\text { Ich exemplarisch vormache, wie die Strategie } \\
\text { anzuwenden ist } \\
\text { S: Meine Mathelehrerin/mein Mathelehrer ... } \\
\text { Erklärt, wie die Lesestrategie angewendet wird } \\
\text { Erklärt, was die Lesestrategie bringt } \\
\text { Macht genau vor/zeigt, wie die neue Lesestrategie } \\
\text { angewendet wird }\end{array}$ & $\alpha=0,87 / 0,84$ \\
\hline $\begin{array}{l}\text { Relevanz } \\
\text { von Lese- } \\
\text { strategien } \\
\text { im Unter- } \\
\text { richt (6) }\end{array}$ & Zustimmung & $\begin{array}{l}\text { L: Ich mache im Mathematikunterricht deutlich, } \\
\text { dass es wichtig ist, ... } \\
\text { S: Meine Mathelehrerin/mein Mathelehrer sagt, } \\
\text { dass es wichtig ist, ... } \\
\text { Lesestrategien beim Verstehen der Aufgabe zu } \\
\text { nutzen } \\
\text { Zu kontrollieren, ob man den Inhalt der } \\
\text { Textaufgabe versteht } \\
\text { Lesestrategien auch in anderen Fächern zu nutzen }\end{array}$ & $\alpha=0,83 / 0,84$ \\
\hline
\end{tabular}

Die Skalierungen beziehen sich auf Häufigkeiten $(1=$ nie oder fast nie/in (fast) keiner Unterrichtsstunde, $2=$ manchmal/in einigen Unterrichtsstunden, 3= oft/in den meisten Unterrichtsstunden, 4= fast immer/in (fast) jeder Unterrichtsstunde) oder Zustimmung ( 1 = stimmt überhaupt nicht, 2 = stimmt eher nicht, $3=$ stimmt eher, $4=$ stimmt ganz genau)

$L$ Lehrkräfte, $S$ Schülerinnen und Schüler 


\subsection{Einschätzung der Vermittlung von Lesestrategien im Fach Mathematik}

Zur Beantwortung der Fragestellungen wurde ein Fragebogen konstruiert. In der Fragebogeninstruktion wurde beiden Personengruppen mündlich und schriftlich erklärt, dass sich die Items auf Unterrichtsstunden beziehen, in denen Textaufgaben bearbeitet werden und es insbesondere um den Einsatz von Lesestrategien für das Verstehen der Textaufgabe geht. Weiter wurde den Lernenden folgender Hinweis gegeben: „Du wirst später oft dem Wort Lesestrategien begegnen. Mit Lesestrategien sind alle Tipps, Methoden oder Schritte gemeint, mit denen du den Inhalt in der Textaufgabe besser verstehen kannst“. Beide Personengruppen wurden zur Häufigkeit der Vermittlung verschiedener kognitiver und metakognitiver Lesestrategiearten beim Bearbeiten von Textaufgaben im Unterricht befragt (vgl. Tab. 2; Boekaerts 1999; Weinstein und Mayer 1986). Bei der Auswahl der kognitiven und metakognitiven Strategiearten wurden solche Lesestrategien aufgeführt, die in der mathematikdidaktischen Forschung als relevant angesehen werden (vgl. Abschnitt 2.2) und es wurden Lesestrategien ergänzt, die für das Textverstehen insgesamt als förderlich gelten (z. B. Zusammenfassen, bildliches Vorstellen; Gold 2018). Mit den Items zur Instruktionsqualität bei der Lesestrategievermittlung wurde erfasst, ob die Lehrkräfte Lesestrategien explizit vermitteln, d.h. ob sie Nutzen und/oder Vorgehen erläutern, die Strategieanwendung modellieren und ihren Lernenden eine eigenständige Anwendung bzw. das Üben von Lesestrategien ermöglichen (Duffy 2002; Duke und Pearson 2002). Abschließend wurde nach der instrumentellen Motivierung zur Nutzung von Lesestrategien im Unterricht bzw. nach der wahrgenommenen Relevanz der Thematik im Unterricht gefragt (vgl. DESI-Studie: Wagner et al. 2009; PISAStudie 2006: Frey et al. 2009).

Die Formulierungen wurden nach einer Pilotierung in zwei fünften Klassen an weiterführenden Schulen für die Lernenden sprachlich angepasst. Die eingesetzten Likert-Skalierungen orientierten sich an Leistungsvergleichsstudien wie DESI (Wagner et al. 2009), IGLU (Bos et al. 2010) und PISA (Frey et al. 2009).

Die Analysen der internen Konsistenz der manifesten Skalen verdeutlichen für beide Personengruppen eine gute Reliabilität.

\subsection{Datenauswertung}

Zur Beantwortung der ersten Fragestellung, wie die Lehrkräfte und Lernenden den Unterricht einschätzen und um zu prüfen, wo Übereinstimmungen und Diskrepanzen vorliegen, wurden basierend auf den fünf Skalenmittelwerten (vgl. Tab. 2) deskriptive Analysen und $t$-Tests mit der Software SPSS (Version 27) durchgeführt. Weiter wurden auch Einzelitems verglichen (vgl. Tab. 6). Zur Beantwortung der zweiten Fragestellung, ob in der Gruppe der Lernenden differenzielle Einschätzungsprofile vorliegen, wurden Latent-Profil-Analysen mit der Software Mplus 8.0 (Muthén und Muthén 2018) durchgeführt, bei denen die Skalenmittelwerte der fünf Einschätzungsskalen als Indikatoren zur Bildung der latenten Profile verwendet wurden. Die Entscheidung über die optimale Anzahl an Profilen wurde sowohl anhand inhaltlicher Kriterien als auch auf Basis absoluter sowie komparativer Modellfitindizes 
getroffen. ${ }^{3}$ Anschließend wurde betrachtet, was die profilspezifischen Substichproben hinsichtlich ihrer Lernvoraussetzungen kennzeichnet. Um differenzielle Wahrnehmungen der Lernendenprofile mit denen der Lehrkräfte zu vergleichen, wurden Varianzanalysen berechnet. Sofern sich keine Varianzhomogenität nachweisen ließ, wurden robuste Testverfahren verwendet (Welch-Anova bzw. für die Post-hoc-Analysen die Games-Howell-Prozedur).

\section{Ergebnisse}

\subsection{Vermittlung von Lesestrategien aus Sicht von Lehrkräften und Lernenden}

Angesichts der ersten Fragestellung, wie die Vermittlung von Lesestrategien aus Sicht von Lehrkräften und Lernenden eingeschätzt wird, werden die Ergebnisse der deskriptiven Analysen sowie $t$-Tests für den Vergleich der Skalenmittelwerte dargestellt (Tab. 3). Eine Übersicht über alle Items, Mittelwerte und Differenzen findet sich in Tab. 6.

Die Vermittlung kognitiver Lesestrategien wird aus Sicht der Lernenden sowie der Lehrpersonen manchmal/in einigen Stunden wahrgenommen. Die Einschätzungen unterscheiden sich auf Gesamtskalenebene nicht, was auf eine geteilte Wahrnehmung hindeutet und die Schlussfolgerung zulässt, dass kognitive Lesestrategien ein Teil des Mathematikunterrichts zu sein scheinen, wenn Textaufgaben bearbeitet werden. Betrachtet man die einzelnen Lesestrategiearten (s. Tab. 6) zeigen sich Über-

Tab. 3 Mittelwerte und Standardabweichungen Einschätzungsskalen von Lernenden und Lehrkräften

\begin{tabular}{|c|c|c|c|c|c|}
\hline \multirow[t]{2}{*}{ Skala } & \multicolumn{2}{|l|}{$M(S D)$} & \multirow[t]{2}{*}{$t$} & \multirow[t]{2}{*}{$D f$} & \multirow[t]{2}{*}{$p$} \\
\hline & $\begin{array}{l}\text { Schülerinnen und } \\
\text { Schüler } \\
(N=261)\end{array}$ & $\begin{array}{l}\text { Lehrkräfte } \\
(N=60)\end{array}$ & & & \\
\hline $\begin{array}{l}\text { Vermittlung kognitiver } \\
\text { Lesestrategiearten }\end{array}$ & $2,35(0,54)$ & $2,37(0,50)$ & $-0,29$ & 318 & 0,772 \\
\hline $\begin{array}{l}\text { Vermittlung metakognitiver } \\
\text { Lesestrategiearten }\end{array}$ & $2,53(0,60)$ & $2,23(0,54)$ & 3,55 & 317 & $<0,001$ \\
\hline $\begin{array}{l}\text { Instruktionsqualität bei der } \\
\text { Lesestrategievermittlung }\end{array}$ & $2,85(0,76)$ & $3,17(0,65)$ & $-2,82$ & 310 & $<0,01$ \\
\hline $\begin{array}{l}\text { Relevanz von Lesestrategien im } \\
\text { Unterricht }\end{array}$ & $2,74(0,65)$ & $2,57(0,60)$ & 1,90 & 314 & 0,058 \\
\hline
\end{tabular}

Die Skalierungen beziehen sich auf Häufigkeiten $(1=$ nie oder fast nie/in (fast) keiner Unterrichtsstunde, $2=$ manchmal/in einigen Unterrichtsstunden, $3=\mathrm{oft} / \mathrm{in}$ den meisten Unterrichtsstunden, 4=fast immer/in (fast) jeder Unterrichtsstunde) oder Zustimmung ( $1=$ stimmt überhaupt nicht, $2=$ stimmt eher nicht, $3=$ stimmt eher, $4=$ stimmt ganz genau)

\footnotetext{
${ }^{3}$ Als Wert des absoluten Modellfits wurde die Entropie herangezogen. Werte nahe 1 weisen auf eine hohe Klassifikationsgenauigkeit hin (Geiser 2010). Bezüglich des komparativen Modellfits wurden neben informationstheoretischen Maßen, wie dem Akaike Informationskriterium und dem Bayesisches Informationskriterium auch inferenzstatistische Maße wie dem Vuong-Lo-Mendell-Rubin-Test und der Lo-MendellRubin adjustierte Likelihood-Ratio-Test zur Modellselektion berücksichtigt. Diese prüfen, ob die Erweiterung des Modells um ein zusätzliches Profil zu einer signifikanten Verbesserung der Datenanpassung führt (Geiser 2010).
} 
einstimmungen beim Markieren und Zusammenfassen, beim Aufstellen von Vermutungen auf der Basis von Bildern, Tabellen und Abbildungen, beim Visualisieren/ Skizzieren, beim Anfertigen von Notizen und beim überfliegenden Lesen. Letzteres wird aus Sicht beider Personengruppen manchmal/in einigen Stunden praktiziert, wohingegen das Zusammenfassen sowie das Aufstellen von Vermutungen mithilfe von Bildern, Tabellen und Abbildungen im Mathematikunterricht oft/in den meisten Stunden zum Einsatz kommen. Unterschiede zwischen den Wahrnehmungen der Lernenden und der Lehrpersonen werden bei Elaborationsstrategien, wie dem bildlichen Vorstellen und dem Formulieren von Hypothesen auf Grundlage von Schlüsselwörtern, ebenso beim wiederholenden Lesen, dem Abschreiben der Textaufgabe und der Betrachtung der Textstruktur ersichtlich. Lernende nehmen das Abschreiben und die Betrachtung der Textstruktur signifikant häufiger wahr als Lehrkräfte, die wiederum eine häufigere Wahrnehmung der anderen benannten Strategien aufweisen (bildliches Vorstellen, Formulieren von Hypothesen auf Grundlage von Schlüsselwörtern und wiederholendes Lesen).

Bei der Vermittlung metakognitiver Lesestrategien sind die Werte der Lernenden signifikant höher als die der Lehrkräfte. Lernende nehmen die entsprechenden Strategien manchmal/in einigen Stunden bis oft/in den meisten Stunden wahr, die Lehrkräfte hingegen geben an, sie manchmal/in einigen Stunden zu vermitteln. Betrachtet man die Einzelitems, so liegen Konvergenzen bei solchen metakognitiven Lesestrategien vor, die sich auf inhaltsbezogene Aspekte des Verstehens der Textaufgabe richten, wie die Verständnisüberwachung und die Kontrolle des inhaltlichen Verstehens. Entsprechende Strategien werden von den Lernenden und den Lehrkräften oft/in den meisten Stunden wahrgenommen und deuten auf vorliegende Unterrichtsroutinen im Mathematikunterricht hin. Eine weitere Gemeinsamkeit betrifft die Strategie, das Verstehens der Textaufgabe als Ziel zu formulieren, die von beiden Personengruppen jedoch nur manchmal/in einigen Stunden des Mathematikunterrichts wahrgenommen wird. Unterschiedlich werden indessen die Planung, Beobachtung und Reflexion des Lesestrategieeinsatzes wahrgenommen. Die Lernenden nehmen den Einsatz dieser metakognitiven Strategien häufiger wahr als die befragten Lehrkräfte.

Bezüglich der Instruktionsqualität bei der Vermittlung von Lesestrategien zeigt sich, dass die Zustimmung zu den Aussagen in den Items bei den Lernenden auf Gesamtskalenebene signifikant geringer ausfällt als bei den Lehrkräften, wenngleich beide Personengruppen den Items im Mittel eher zustimmen und alle Items im Vergleich mit den anderen Skalen auf recht hohem Niveau einschätzen. Bei näherer Betrachtung der einzelnen Items zeigt sich, dass sich die Wahrnehmungen der Erklärung der Anwendung von Lesestrategien, der Erläuterung des Nutzens, der Modellierung der Anwendung sowie der Anleitung zum gemeinsamen Üben zwischen Lernenden und Lehrkräften nicht unterscheidet. Lediglich beim eigenständigen Üben von Lesestrategien fällt die Zustimmung der Lernenden signifikant geringer aus (stimmen eher nicht bis eher zu) als die der Lehrkräfte, die diesem Item eher zustimmen.

Den Items zur Relevanz von Lesestrategien im Mathematikunterricht stimmen beide Personengruppen ohne bedeutsamen Unterschied mäßig zu. Dass Lesestrategien für das Verstehen von Textaufgaben, aber auch in anderen Fächern bedeutsam 
sind, wird von Lernenden und Lehrkräften als zutreffend betrachtet. Unterschiedliche Einschätzungen zeigen sich bei den Items mit den Aussagen zur Planung des Lesestrategieeinsatzes, zur Auswahl passender Lesestrategien, zur Bewertung des strategischen Vorgehens sowie zur Kontrolle des Textverständnisses, wobei Lernende ausschließlich dem letztgenannten Aspekt signifikant weniger zustimmen als die Lehrkräfte.

\subsection{Differenzielle Einschätzungen des Unterrichts aus Sicht von Lernenden}

Um differenzielle Einschätzungen der Vermittlung von Lesestrategien im Unterricht in der Gruppe der Lernenden zu prüfen, wurden latente Profilanalysen auf Grundlage ihrer Einschätzungswerte durchgeführt (Tab. 4).

Die informationstheoretischen Maße AIC und BIC nehmen mit steigender Anzahl an Profilen ab, was auf eine Lösung mit einer möglichst hohen Profilzahl hindeutet, jedoch dem Gebot der Sparsamkeit widerspricht. Die Ergebnisse des VLMR- und des LMRA-Tests zeigen zudem, dass sich die Datenstruktur der Lernenden durch zwei Profile sinnvoll abbilden lässt und auch die Klassifikationsgenauigkeit mit einer Entropie von0,97 für eine Lösung mit zwei Profilen gut ausfällt. Da die Hinzunahme eines weiteren Profils nicht zu einer signifikanten Verbesserung der Modellanpassung führt $(p=0,58)$ und die 2-Profil-Lösung signifikant besser ist $(p=0,03)$ als ein Berechnung ohne Profile, wird die 2-Profil-Lösung für die weiteren Analysen verwendet. Jeweils beide Profile sind in jeder der einbezogenen Schulklassen vertreten. Tab. 5 verdeutlicht die deskriptiven Statistiken und Ergebnisse der Varianzanalysen, bei welchen die Gruppenzugehörigkeit (Profil 1, Profil 2, Lehrkräfte) als unabhängige Variable und die Mittelwerte der Einschätzungsskalen (linke Spalte) als abhängige Variablen eingingen. Abb. 1 veranschaulicht zudem die Mittelwerte beider Lernendenprofile und die der Lehrkräfte.

Zunächst die deskriptiven Einschätzungswerte der Lernenden innerhalb der Profile betrachtend (mittlere Spalten in Tab. 5), zeichnet sich Profil 1 (52\% der Lernenden) durch eine manchmal/in einigen Stunden bzw. oft/in den meisten Stunden wahrgenommene kognitive bzw. metakognitive Strategievermittlung aus. Diese Lernenden stimmen eher zu, dass ihre Lehrkräfte Lesestrategien explizit vermitteln und diese Thematik im Unterricht betonen. Profil 2 repräsentiert Lernende (48\%), welche die Vermittlung der kognitiven und metakognitiven Lesestrategien im Unterricht manchmal/in einigen Stunden wahrnehmen. Sie stimmen eher nicht bis eher den Items der Instruktionsqualität und der Relevanz im Unterricht zu. Post-hoc-

Tab. 4 Profilanalysen basierend auf Einschätzungsmittelwerten der Lernenden

\begin{tabular}{lllllllll}
\hline Profile & AIC & BIC & Entropie & $p$ & $p$ & \multicolumn{2}{l}{ Klassengrößen (\%) } & \\
& & & & (VLMRT) & $($ LMRAT) & Profil 1 & Profil 2 & Profil 3 \\
\hline 1 & 2742,35 & 2777,99 & - & - & - & 261 & - & - \\
2 & & & & & & $(100)$ & & \\
3 & 2508,55 & 2565,58 & 0,971 & 0,03 & 0,03 & $136(52)$ & $125(48)$ & - \\
\hline
\end{tabular}

AIC Akaike Informationskriterium, BIC Bayesisches Informationskriterium, VLMRT Vuong-Lo-MendellRubin-Test, LMRAT Lo-Mendell-Rubin adjustierte Likelihood-Ratio-Test 
Tab. 5 Mittelwerte und Standardabweichungen der Einschätzungsskalen von Lernendenprofilen und Lehrkräften

\begin{tabular}{|c|c|c|c|c|}
\hline \multirow[b]{2}{*}{ Skala } & \multicolumn{3}{|c|}{$\begin{array}{l}\text { Deskriptive Statistiken } \\
M(S D)\end{array}$} & \multirow[t]{2}{*}{ ANOVA } \\
\hline & $\begin{array}{l}\text { Profil } 1 \\
(N=136)\end{array}$ & $\begin{array}{l}\text { Profil 2 } \\
(N=125)\end{array}$ & $\begin{array}{l}\text { Lehrkräfte } \\
(N=60)\end{array}$ & \\
\hline $\begin{array}{l}\text { Vermittlung } \\
\text { kognitiver } \\
\text { Lesestrate- } \\
\text { giearten }\end{array}$ & $2,61(0,47)$ & $2,06(0,45)$ & $2,37(0,50)$ & $\begin{array}{l}F(2,319)=45,42, p<0,001, \eta^{2}=0,22 \\
\text { P1 vs. } \mathrm{P} 2 \text { vs. } \mathrm{L}, p<0,001\end{array}$ \\
\hline $\begin{array}{l}\text { Vermittlung } \\
\text { meta- } \\
\text { kognitiver } \\
\text { Lesestrate- } \\
\text { giearten }\end{array}$ & $2,99(0,41)$ & $2,04(0,33)$ & $2,23(0,54)$ & $\begin{array}{l}F(2,318)=187,68, p<0,001, \eta^{2}=0,54 \\
\text { P1 vs. } \mathrm{P} 2, p<0,001 \\
\text { P1 vs. } \mathrm{L}, p<0,001 \\
\mathrm{P} 2 \text { vs. } \mathrm{L}, p<0,05\end{array}$ \\
\hline $\begin{array}{l}\text { Instruktions- } \\
\text { qualität bei } \\
\text { der Lesestra- } \\
\text { tegievermitt- } \\
\text { lung }\end{array}$ & $3,15(0,58)$ & $2,52(0,81)$ & $3,17(0,65)$ & $\begin{array}{l}F(2,311)=32,05, p<0,001, \eta^{2}=0,17 \\
\mathrm{P} 1 \text { vs. } \mathrm{P} 2, p<0,001 \\
\mathrm{P} 2 \text { vs. } \mathrm{L}, p<0,001\end{array}$ \\
\hline $\begin{array}{l}\text { Relevanz } \\
\text { von Lese- } \\
\text { strategien im } \\
\text { Unterricht }\end{array}$ & $3,03(0,53)$ & $2,43(0,64)$ & $2,57(0,60)$ & $\begin{array}{l}F(2,315)=36,14, p<0,001, \eta^{2}=0,19 \\
\mathrm{P} 1 \text { vs. } \mathrm{P} 2, p<0,001 \\
\mathrm{P} 1 \text { vs. } \mathrm{L}, p<0,001\end{array}$ \\
\hline
\end{tabular}

Die Skalierungen bezogen sich auf Häufigkeiten $(1=$ nie oder fast nie/in (fast) keiner Unterrichtsstunde, $2=$ manchmal/in einigen Unterrichtsstunden, $3=$ oft $/$ in den meisten Unterrichtsstunden, $4=$ fast immer/in (fast) jeder Unterrichtsstunde) oder Zustimmung ( $1=$ stimmt überhaupt nicht, $2=$ stimmt eher nicht, $3=$ stimmt eher, $4=$ stimmt ganz genau)

$P 1$ Lernende in Profil 1, $P 2$ Lernende in Profil 2, $L$ Lehrkräfte

Tests zeigen, dass sich die Einschätzungen profilbezogen unterscheiden (vgl. rechte Spalte P1 vs. P2).

Um näher zu betrachten, worin sich die Lernenden hinsichtlich kognitiver Lernvoraussetzungen unterscheiden, wurden die jeweiligen Hintergrundvariablen verglichen. Es zeigt sich, dass beide Profile ein ausgeglichenes Verhältnis bezüglich des Geschlechts (Profil 1; 51,2\% weiblich; Profil 2: 48,8\% weiblich) und der Muttersprache (Profil 1: 73,7\% Deutsch als Muttersprache; Profil 2: 76,0\% Deutsch als Muttersprache) aufweisen. Auch bezogen auf die Fachnoten in Deutsch und Mathematik lassen sich keine Unterschiede ermitteln (Profil 1: Deutschnote $M=2,74, S D=0,56$, Mathenote: $M=2,71, S D=0,93$; Profil 2: Deutschnote: $M=2,69, S D=0,80$, Mathenote: $M=2,65, S D=0,86)$. Mit Blick auf die kognitiven Lernvoraussetzungen ergibt sich, dass sich die Lernenden nicht in ihrer kognitiven Grundfähigkeit, der Leseflüssigkeit und dem Lesestrategiewissen unterscheiden, aber in ihrem Leseverständnis $(t(259)=-2,37, p<0,05)$ : Lernende in Profil 1 haben ein signifikant geringes Leseverständnis $(M=9,86, S D=3,69)$ als die Lernenden in Profil $2(M=10,91, S D=3,46)$. Demnach nehmen die Lernenden mit geringeren Werten im Leseverständnistest (Profil 1) den Mathematikunterricht als durchweg strategieorientierter wahr als die besseren Leserinnen und Leser (Profil 2). 


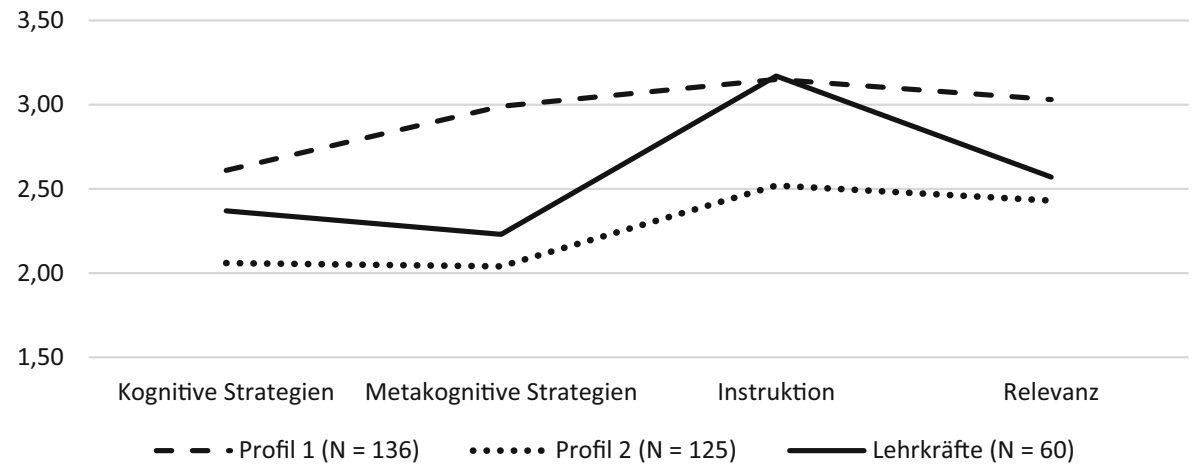

Abb. 1 Mittlere Einschätzungen der Strategievermittlung beider Lernendenprofile und der Lehrkräfte

Werden die Einschätzungen der beiden Lernendenprofile mit denen der Lehrkräfte verglichen (rechte Spalte in Tab. 5 sowie Abb. 1), so zeigt die Anova, dass die Lernenden mit schlechterem Leseverständnis in Profil 1 bis auf die explizite Instruktion von Lesestrategien in allen Einschätzungswerten von den Einschätzungen der Lehrkräfte signifikant abweichen und ein höheres Maß an Strategievermittlung und Relevanzsetzung wahrnehmen. Lernende aus Profil 2 hingegen nehmen signifikant weniger kognitive und metakognitive Lesestrategien und Instruktionsqualität wahr; lediglich die Relevanz schätzen sie vergleichbar ein wie ihre Lehrkräfte. Die größten Unterschiede werden insgesamt bei der Wahrnehmung der Vermittlung metakognitiver Lesestrategien ersichtlich.

\section{Diskussion}

Die Studie bietet einen ersten Einblick, ob Lesestrategien im üblichen Mathematikunterricht eine Rolle spielen, wenn Textaufgaben bearbeitet werden. Ergebnis der Studie ist, dass zunächst ohne Berücksichtigung von lernendenseitigen Lernvoraussetzungen kognitive Lesestrategien in Stunden, in denen Textaufgaben bearbeitet werden, von Lehrkräften und Lernenden manchmal/in einigen Stunden wahrgenommen werden. Vor allem bei den Items zu den Organisationsstrategien, die auch im Fokus der Forschung stehen und von dieser als förderlich für das Verstehen von Textaufgaben eingeschätzt werden (Hagena et al. 2017; Prediger und Krägeloh 2015; Schukajlow und Leiss 2011), stimmen die Lehrkräfte und Lernenden überein. Bei einigen Elaborationsstrategien hingegen fallen die Angaben der Lernenden im Vergleich mit den Lehrkräften signifikant geringer aus, wie etwa beim bildlichen Vorstellen oder dem Formulieren von Hypothesen auf Grundlage von Schlüsselwörtern. Denkbar ist, dass die geringere Wahrnehmung der letztgenannten elaborierenden Strategiearten aufseiten der Lernenden damit zusammenhängt, dass Elaborationsstrategien im Unterricht von den Lehrkräften (nebenbei) mündlich eingefordert werden, wohingegen Organisationsstrategien in Form von Lesestrategie-Techniken in schriftliche Handlungen münden, und deshalb vermutlich wahrnehmungsauffälliger sind. Angesichts der metakognitiven Lesestrategien zeigt sich, 


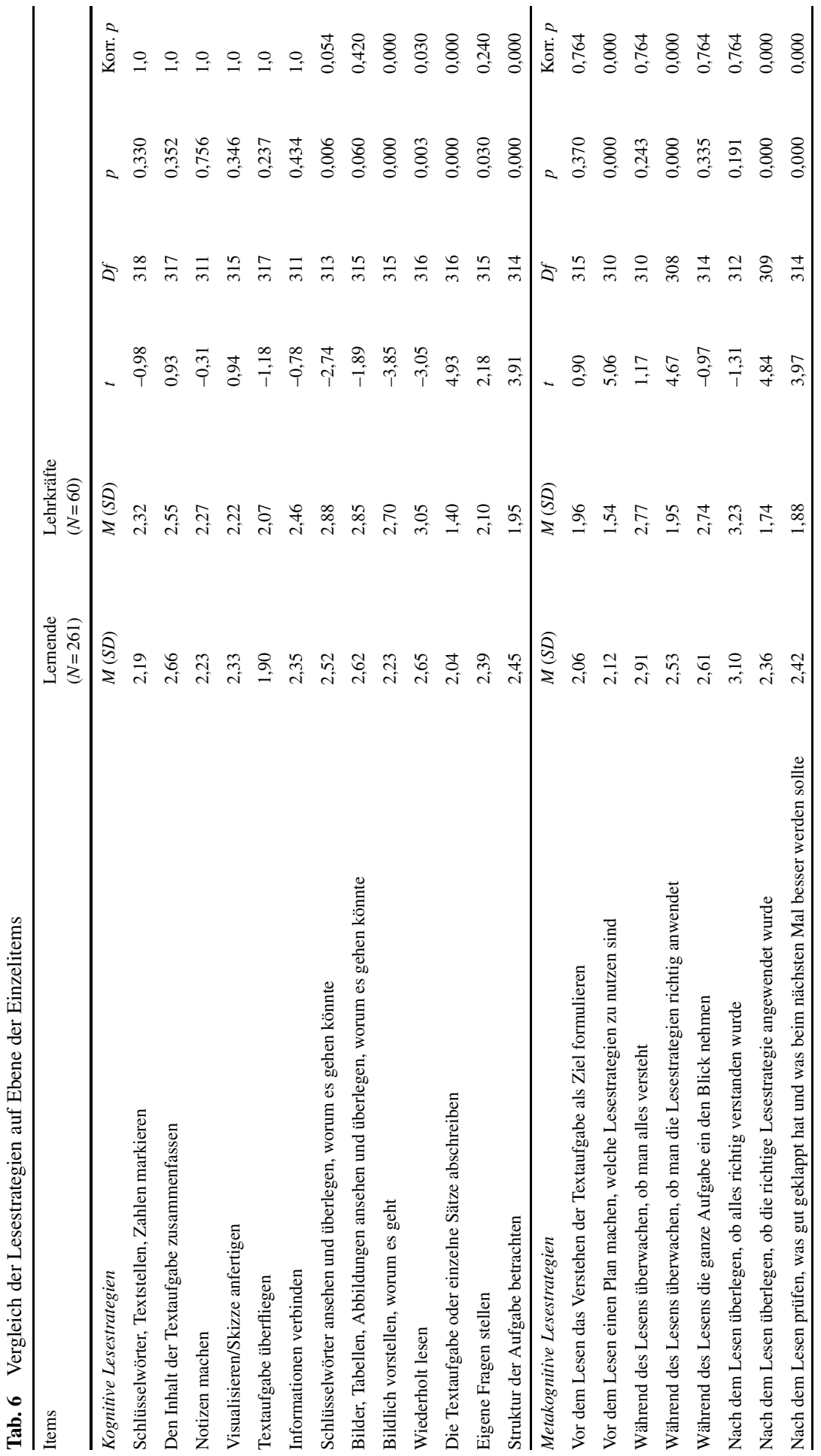




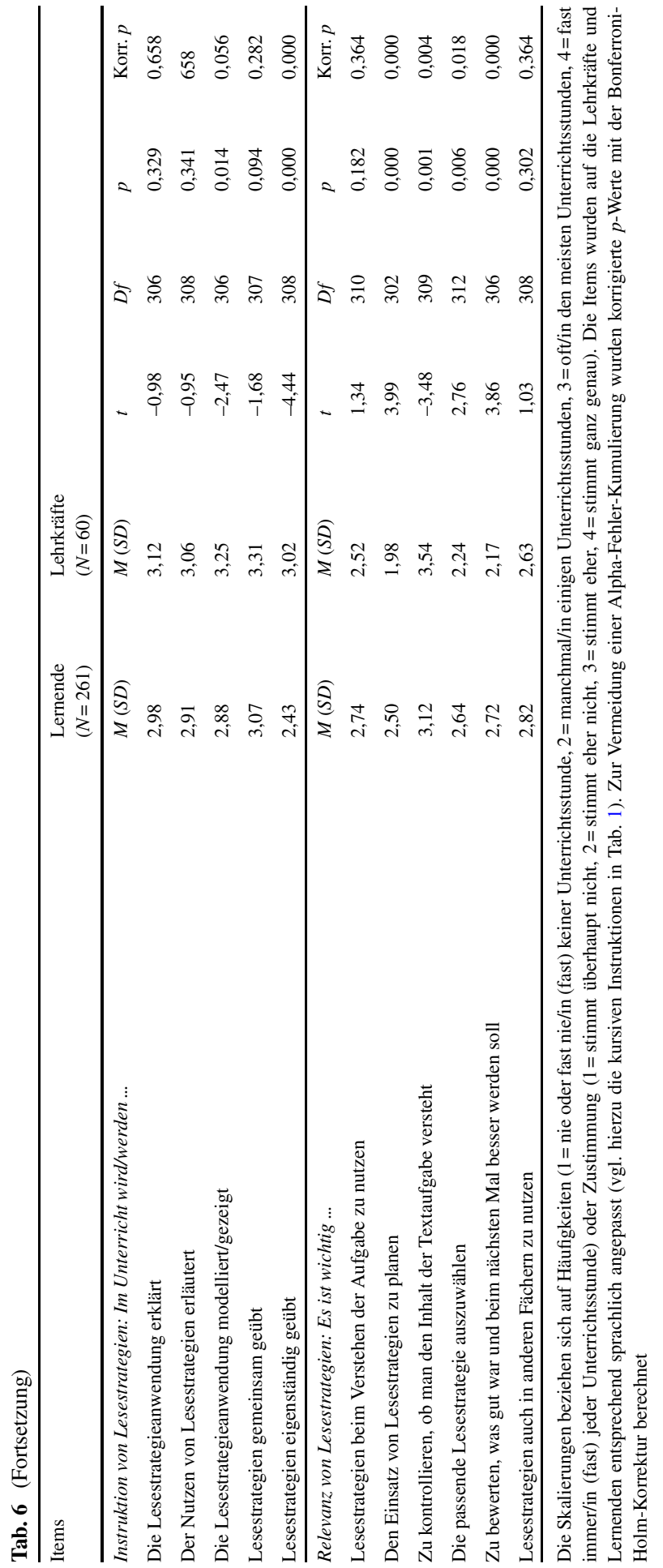


dass diese vergleichbar oft wie kognitive Lesestrategien wahrgenommen werden, die Lernenden aber im Gegensatz zu den Lehrkräften von einer häufigeren Instruktion metakognitiver Lesestrategien berichten. Den Angaben der Lernenden sowie der Lehrkräfte zufolge scheinen Strategien, die sich auf das inhaltliche Verstehen von Textaufgaben beziehen, häufiger stattzufinden als strategiebezogene metakognitiven Facetten, die auch in Selbstberichten von Lernenden bei Schukajlow und Leiss (2011) eine Randstellung einnehmen. Bei der Instruktion von Lesestrategien wird evident, dass die Lernenden die Qualität der Strategieinstruktion zwar signifikant geringer einschätzen als die Lehrpersonen, aber trotz dessen eher den Aussagen in den Items zustimmen. Dies widerspricht dem Befund von Dignath und Büttner (2018), die eine eher implizite Vermittlungspraxis bei den Mathematiklehrkräften das selbstregulierte Lernen betreffend beobachteten. Bei der instrumentellen Motivierung bzw. der wahrgenommenen Relevanz der Thematik im Unterricht sind sich die Persongruppen einig, dass die Lehrkräfte den Stellenwert von Lesestrategien in ihrem Unterricht mäßig betonen.

Die personenzentrierten Profilanalysen verdeutlichen darüber hinaus, dass die Wahrnehmung der Strategievermittlung im Unterricht von kognitiven Lernvoraussetzungen, dem Leseverständnis, abhängt und die Lernenden den Unterricht innerhalb von Klassen differenziell einschätzen (Jurik et al. 2015; Seidel 2006). Wie bei Holzberger et al. (2016) unterscheiden sich ihre Einschätzungen zu den Items vor allem in der Höhe der Ausprägungen (ähnliche Befunde zeigen sich bei Vieluf et al. 2012; Schiepe-Tiska et al. 2013). Schwächere Leserinnen und Leser schätzen den Mathematikunterricht als grundsätzlich lesestrategieorientierter ein als die besseren Rezipientinnen und Rezipienten. Auffällig ist darunter das Ergebnis, dass die schlechteren Leserinnen und Leser deutlich mehr metakognitive Lesestrategien wahrnehmen. Bei der Instruktion von Lesestrategien sowie der instrumentellen Motivierung bestehen Konvergenzen zwischen den Einschätzungen einer der beiden Lernendenprofile und den Angaben der Lehrkräfte. Dass zwischen der Lernendenund der Lehrkraftperspektive allenfalls moderate Übereinstimmungen anzunehmen sind (Clausen 2002; Fauth et al. 2020), scheint somit nur in Teilen zutreffend, wenn die Lernvoraussetzungen der Lernenden in die Analysen einbezogen werden.

Die Vermittlung von Lesestrategien ist in den betrachteten Schulen insbesondere für die schwächeren Leserinnen und Leser wahrnehmungsauffällig. Da es aber für alle Lernenden laut Leseforschung in diesem Alter positiv ist, wenn sie eine zielgerichtete Vermittlung verschiedener Strategiearten bewusst erfahren und deren Anwendung wiederholt einüben (Gold 2018), wäre es wünschenswert, wenn verschiedene kognitive Lesestrategiearten, insbesondere solche, die von der mathematikdidaktischen Forschung als wirksam eingeordnet werden (Hagena et al. 2017; Schukajlow und Leiss 2011; Prediger und Krägeloh 2015), für alle Lernenden expliziert würden. Vor dem Hintergrund der nachgewiesenen Schwierigkeiten von Lernenden bei der zielgerichteten Strategieanwendung (Böckmann und Schukajlow 2020), wäre eine explizite Vermittlungspraxis wünschenswert, die auch die Modellierung der Strategieanwendung umfasst. Auf diese Weise würden die Lernenden Lesestrategien zum Verstehen des Aufgabentextes zielgerichtet anwenden lernen, wodurch sich dem Mediations- sowie Nutzungsdefizit oder trägem Wissen, wie in der letzten PISA-Kohorte verdeutlicht, entgegenwirken ließe (Diedrich et al. 2019; 
Hasselhorn 1996). Neben Organisationsstrategien geht es dabei auch um Elaborationsstrategien, da sie die Konstruktion des Situationsmodells in besonderem Maße begünstigen (Gold 2018). Ferner wäre eine Berücksichtigung strategiebezogener metakognitive Prozesse, wie die Planung und Überwachung des Lesestrategieeinsatzes sowie die Reflexion über das strategische Vorgehen, zu nennen. Solche metakognitiven Aktivitäten fallen Lernenden zwar besonders schwer (Alexander et al. 1995), eine frühe Vermittlung kann jedoch zu einer langfristigen Automatisierung sowie nachhaltigen Effekten auf das Textverstehen beitragen.

Wenngleich die vorliegende Studie keine Antworten auf Ursachen für die Antworttendenzen der beteiligten Personen (-gruppen) bieten kann, sollen an dieser Stelle Erklärungsansätze für die Niveauunterschiede der Wahrnehmung auf Seiten der Lernenden angeführt werden. Dass Lernende mit schlechteren Leseverständniswerten die Lesestrategievermittlung bei der Bearbeitung von Textaufgaben eher wahrnehmen, könnte damit zusammenhängen, dass sie diese als hilfreiches Mittel erachten, oder aber der Lesestrategieeinsatz sie vor zusätzliche kognitive Herausforderungen stellt (Hasselhorn 1996) und er womöglich nicht ihrem üblichen Vorgehen entspricht (Dröse und Prediger 2019; Prediger und Krägeloh 2015; Verschaffel et al. 2000). Metakognitive Lesestrategien nehmen sie vermutlich aufgrund ihres besonderen Anspruchs (Alexander et al. 1995) vermehrt wahr. Dass die besseren Leserinnen und Leser die strategischen Hilfen für die Erschließung von Textaufgaben weniger wahrnehmen, könnte wiederum damit zusammenhängen, dass sie mit dem Verstehen des Aufgabentextes grundsätzlich weniger Schwierigkeiten haben und daher bereits auf den Lösungsprozess fokussiert sind. Um die selektiven Wahrnehmungen und auch die Bedürfnisse der Lernenden näher zu untersuchen, bedarf es ergänzender qualitativer Studiendesigns (z.B. Interview- und Beobachtungsstudien). In diesem Zusammenhang wäre es sinnvoll, neben den in dieser Studie erfassten lesebezogenen Lernvoraussetzungen auch mathematikspezifische Leistungsmerkmale der Lernenden zu erheben, um eine umfassendere und vor allem auch fachspezifischere Berücksichtigung von Bedürfnissen und Leistungsständen der Lernenden zu gewährleisten. Weiter zeigt der aktuelle Forschungsstand, dass die verschiedenen Lesestrategiearten von der mathematikdidaktischen Forschung zukünftig verstärkt untersucht und ihr spezifischer Beitrag für die Konstruktion des Situationsmodells ermittelt werden sollte (s. bspw. Böckmann und Schukajlow 2020).

Abschließend sollen Limitationen der Studie diskutiert werden. Angesichts der einbezogenen Stichproben ist darauf hinzuweisen, dass sie aus Schulen stammten, die mit ihrer Teilnahme am BiSS-Programm für das Thema Leseförderung im Mathematikunterricht sensibilisiert wurden. Beobachtungsstudien in einem Teil der Schulen verdeutlichen diesbezüglich, dass die Auseinandersetzung mit Textaufgaben im Mathematikunterricht an diesen Schulen tendenziell etwas strategieorientierter erfolgte als im Mathematikunterricht an Kontrollschulen (Schmitz et al. 2021). Des Weiteren sind methodische Einschränkungen zu nennen, die mit der Durchführung von Fragebogenstudien einhergehen. Diesbezüglich ist einerseits auf mögliche Verzerrungen wie Milde-Strenge-Tendenzen oder die soziale Erwünschtheit zu verweisen und andererseits zu erwähnen, dass nicht abschließend beurteilt werden kann, ob die Lernenden und Lehrkräfte bei der Beantwortung des Fragebogens trotz vorhergehender mündlicher und schriftlicher Instruktion nur die spezifische Situation 
des Verstehens von Textaufgaben vor Augen hatten. Ferner ermöglichen die mehrperspektivischen Befragungen und die geteilten Wahrnehmungen zwar einen umfassenden, aber nur einen indirekten Einblick in das Unterrichtshandeln (Baumert et al. 2004; Maaß und Engeln 2008). Um erweitere Einblicke zu erhalten und die Perspektiven von Lehrkräften und Lernenden zu ergänzen, bieten sich zusätzliche qualitative Erhebungen mittels Videografie oder Beobachtung an. Jedoch wäre hierbei zu beachten, dass der Beurteilungszeitraum entsprechend angepasst wird, indem bspw. sowohl die Befragung als auch Beobachtung sich auf dieselbe Stunde richten, was wiederum dazu führt, dass keine Langzeiterfahrungen und Unterrichtsroutinen im Mittelpunkt stehen, die in der vorliegenden Studie von Interesse waren. Eine klassenbasierte Zuordnung der befragten Lernenden zu ihren spezifischen Lehrkräften, die in der aktuellen Studie aufgrund von strengen Datenschutzbestimmungen leider nicht erfolgen konnte, würde zukünftig ebenfalls eine wünschenswerte Erweiterung darstellen.

Funding Open Access funding enabled and organized by Projekt DEAL.

Open Access Dieser Artikel wird unter der Creative Commons Namensnennung 4.0 International Lizenz veröffentlicht, welche die Nutzung, Vervielfältigung, Bearbeitung, Verbreitung und Wiedergabe in jeglichem Medium und Format erlaubt, sofern Sie den/die ursprünglichen Autor(en) und die Quelle ordnungsgemäß nennen, einen Link zur Creative Commons Lizenz beifügen und angeben, ob Änderungen vorgenommen wurden.

Die in diesem Artikel enthaltenen Bilder und sonstiges Drittmaterial unterliegen ebenfalls der genannten Creative Commons Lizenz, sofern sich aus der Abbildungslegende nichts anderes ergibt. Sofern das betreffende Material nicht unter der genannten Creative Commons Lizenz steht und die betreffende Handlung nicht nach gesetzlichen Vorschriften erlaubt ist, ist für die oben aufgeführten Weiterverwendungen des Materials die Einwilligung des jeweiligen Rechteinhabers einzuholen.

Weitere Details zur Lizenz entnehmen Sie bitte der Lizenzinformation auf http://creativecommons.org/ licenses/by/4.0/deed.de.

\section{Literatur}

Alexander, P. A., Carr, M., \& Schwanenflugel, P. J. (1995). Development of metacognition in gifted children: directions for future research. Developmental Review, 15, 1-37. https://doi.org/10.1037/00121649.30.5.709.

Baumert, J., Kunter, M., Brunner, M., Krauss, S., Blum, W., \& Neubrand, M. (2004). Mathematikunterricht aus Sicht der PISA-Schülerinnen und Schüler und ihrer Lehrkräfte. In P.-K. Deutschland (Hrsg.), PISA 2003 - Der Bildungsstand der Jugendlichen in Deutschland - Ergebnisse des zweiten internationalen Vergleichs (S. 314-354). Münster: Waxmann. https://doi.org/10.1007/s11618-005-0156-8.

Berger, J.-L., \& Karabenick, S. A. (2011). Motivation and students' use of learning strategies: evidence of unidirectional effects in mathematics classrooms. Learning and Instruction, 21, 416-428. https://doi. org/10.1016/j.learninstruc.2010.06.002.

Bescherer, C., \& Papadopoulou, P. (2017). (Sprach-) Förderung beim Bearbeiten von Text- und Sachaufgaben im Mathematikunterricht der Sekundarstufe I. In D. Leiss, A. Neumann \& K. Schwippert (Hrsg.), Mathematik und Sprache. Empirischer Forschungsstand und unterrichtliche Herausforderungen (S. 127-146). Münster: Waxmann.

Blum, W., \& Leiss, D. (2005). Modellieren im Unterricht mit der „Tanken“-Aufgabe. Mathematik lehren, $128,18-21$.

Böckmann, M., \& Schukajlow, S. (2020). Bewertung der Teilkompetenzen Verstehen und Vereinfachen/ Strukturieren und ihre Relevanz für das mathematische Modellieren. In G. Greefrath \& K. Maaß 
(Hrsg.), Modellierungskompetenzen - Beurteilung und Bewertung (S. 113-131). Wiesbaden u.a.: Springer.

Boekaerts, M. (1999). Self-regulated learning: where we are today. International Journal of Educational Research, 31, 445-457.

Bos, W., Strietholt, R., Goy, M., Stubbe, T. C., Tarelli, I., \& Hornberg, S. (2010). IGLU 2006. Dokumentation der Erhebungsinstrumente. Münster u. a.: Waxmann.

Clausen, M. (2002). Unterrichtsqualität - eine Frage der Perspektive. Empirische Analysen zur Übereinstimmungs-, Konstrukt- und Kriteriumsvalidität. Münster: Waxmann.

Cohors-Fresenborg, E., \& Kaune, C. (2003). Unterrichtsqualität: Die Rolle von Diskursivität für „guten“ gymnasialen Mathematikunterricht. Beiträge zum Mathematikunterricht, 173-180.

Cromley, J.G., \& Azevedo, R. (2007). Testing and refining the direct and inferential mediation model of reading comprehension. Journal of Educational Psychology, 99(2), 311-325. https://doi.org/10.1037/ 0022-0663.99.2.311.

De Jong, R., \& Westerhof, K. J. (2001). The quality of student ratings of teacher behavior. Learning Environments Research, 4, 51-85. https://doi.org/10.1023/A:1011402608575.

Diedrich, J., Schiepe-Tiska, A., Ziernwald, L., Tupac-Yupanqui, A., Weis, M., McElvany, N., \& Reiss, K. (2019). Lesebezogene Schülermerkmale in PISA 2018: Motivation, Leseverhalten, Selbstkonzept und Lesestrategiewissen. In K. Reiss, M. Weis, E. Klieme \& O. Köller (Hrsg.), PISA 2018. Grundbildung im internationalen Vergleich (S. 81-110). Münster: Waxmann.

Dignath, C., \& Büttner, G. (2008). Components of fostering self-regulated learning among students. A meta-analysis on intervention studies at primary and secondary school level. Metacognition and Learning, 3(3), 231-264. https://doi.org/10.1007/s11409-008-9029-x.

Dignath, C., \& Büttner, G. (2018). Teachers' direct and indirect promotion of self-regulated learning in primary and secondary school mathematics classes-insights from video-based classroom observations and teacher interviews. Metacognition and Learning, 13, 127-157. https://doi.org/10.1007/s11409018-9181-X.

Ditton, H. (2002). Lehrkräfte und Unterricht aus Schülersicht. Ergebnisse einer Untersuchung im Fach Mathematik. Zeitschrift für Pädagogik, 48(2), 262-286.

Dröse, J. (2020). Textaufgaben lesen und verstehen lernen. Entwicklungsforschungsstudie zur mathematikspezifischen Leseverständnisförderung. Dortmunder Beiträge zur Entwicklung und Erforschung des Mathematikunterrichts, Bd. 43. Wiesbaden: Springer Spektrum.

Dröse, J., \& Prediger, S. (2019). Scaffolding für fachbezogene textsorten-spezifische Lesestrategien - Entwicklungsforschungsstudie zur Förderung des Umgangs mit Textaufgaben. In B. Ahrenholz, S. Jeuk, B. Lütke, J. Paetsch \& H. Roll (Hrsg.), Fachunterricht, Sprachbildung und Sprachkompetenzen (S. 107-134). Berlin: De Gruyter.

Duffy, G. G. (2002). The case for direct explanation of strategies. Comprehension instruction. Researchbased best practices. In C.C. Block \& M. Pressley (Hrsg.), Comprehension instruction. Researchbased best practices (S. 28-41). New York, London: Guilford.

Duke, N.K., \& Pearson, P.D. (2002). Effective practices for developing reading comprehension. In A.E. Farstrup \& S.J. Samuels (Hrsg.), What research has to say about reading instruction (S. 205-242). Newark: International Reading Association.

Erath, K., Ingram, J., Moschkovich, J., \& Prediger, S. (2021). Designing and enacting instruction that enhances language for mathematics learning: a review of the state of development and research. ZDM-Mathematics Education, 53, 245-262. https://doi.org/10.1007/s11858-020-01213-2.

Fauth, B., Decristan, J., Rieser, S., Klieme, E., \& Büttner, G. (2014). Student ratings of teaching quality in primary school: dimensions and prediction of student outcomes. Learning and Instruction, 29, 1-9. https://doi.org/10.1016/j.learninstruc.2013.07.001.

Fauth, B., Göllner, R., Lenske, G., Praetorius, A.-K., \& Wagner, W. (2020). Who sees what? Conceptual considerations on the measurement of teaching quality from different perspectives. Zeitschrift für Pädagogik, 66, 138-155. Beiheft 1/20.

Frey, A., et al. (2009). PISA 2006 Skalenhandbuch. Dokumentation der Erhebungsinstrumente. Münster u. a.: Waxmann.

Geiser, C. (2010). Datenanalyse mit Mplus: Eine anwendungsorientierte Einführung. Wiesbaden: Springer VS.

Gold, A. (2018). Lesen kann man lernen. Wie man die Lesekompetenz fördern kann. Göttingen: Vandenhoeck \& Ruprecht.

Hagena, M., Leiss, D., \& Schwippert, K. (2017). Using reading strategy training to foster students' mathematical modelling competencies: results of a quasi-experimental control trial. Eurasia Journal of Ma- 
thematics, Science and Technology Education, 13(7), 4057-4085. https://doi.org/10.12973/eurasia. 2017.00803a.

Hamman, D., Berthelot, J., Saia, J., \& Crowley, E. (2000). Teachers' coaching of learning and its relation to students' strategic learning. Journal of Educational Psychology, 92(2), 342-348. https://doi.org/ 10.1037/0022-0663.92.2.342.

Hasselhorn, M. (1996). Kategoriales Organisieren bei Kindern. Göttingen: Hogrefe.

Heinze, A., Herwartz-Emden, L., Braun, C., \& Reiss, K. (2011). Die Rolle von Kenntnissen der Unterrichtssprache beim Mathematiklernen: Ergebnisse einer quantitativen Längsschnittstudie in der Grundschule. In S. Prediger \& E. Özdil (Hrsg.), Mathematiklernen unter Bedingungen der Mehrsprachigkeit. Stand und Perspektiven der Forschung und Entwicklung in Deutschland (Bd. 32, S. 11-33). Münster: Waxmann.

Helmke, A. (2017). Unterrichtsqualität und Lehrerprofessionalität. Diagnose, Evaluation und Verbesserung des Unterrichts. Seelze-Velber: Kallmeyer.

Holzberger, D., Kunter, M., Praetorius, A.-K., \& Seidel, T. (2016). Individuelle Schwerpunkte im Mathematikunterricht? Eine latente Profilanalyse zu unterschiedlichen Mustern der Unterrichtsqualität. In N. McElvany, W. Bos, H. G. Holtappels, M. M. Gebauer \& F. Schwabe (Hrsg.), Bedingungen und Effekte guten Unterrichts (S. 135-146). Münster, New York: Waxmann.

Jurik, V., Häusler, J., Stubben, S., \& Seidel, T. (2015). Interaction. Erste Ergebnisse einer vergleichenden Videostudie im Deutsch- und Mathematikunterricht. Zeitschrift für Pädagogik, 61(5), 692-711. https://doi.org/10.25656/01:15422.

Kintsch, W. (1998). Comprehension: a paradigm for cognition. Cambridge: Cambridge University Press.

Kintsch, W., \& Greeno, J. G. (1985). Understanding and solving word arithmetic problems. Psychological Review, 92(1), 109-129. https://doi.org/10.1037/0033-295X.92.1.109.

Kistner, S., Rakoczy, K., Otto, B., Dignath-van Ewijk, C., Büttner, G., \& Klieme, E. (2010). Promotion of self-regulated learning in classrooms: investigating frequency, quality, and consequences for student performance. Metacognition and Learning, 5(2), 157-171. https://doi.org/10.1007/s11409-0109055-3.

Kistner, S., Rakoczy, K., Otto, B., Klieme, E., \& Büttner, G. (2015). Teaching learning strategies: the role of instructional context and teacher beliefs. Journal for Educational Research Online/journal Für Bildungsforschung Online, 7(1), 176-197.

Klieme, E., Pauli, C., \& Reusser, K. (2009). The pythagoras study: Investigating effects of teaching and learning in Swiss and German mathematics classrooms. In T. Janik \& T. Seidel (Hrsg.), The power of video studies in investigating teaching and learning in the classroom (S. 137-160). Münster: Waxmann.

Krug, A., \& Schukajlow, S. (2012). Offene Aufgaben: Schülereinstellungen und Teilaktivitäten beim Modellieren. In M. Ludwig \& M. Kleine (Hrsg.), Beiträge zum Mathematikunterricht (S. 481-484). Münster: WTM.

Leiss, D., \& Plath, J. (2020). „Im Mathematikunterricht muss man auch mit Sprache rechnen!“ - Sprachbezogene Fachleistung und Unterrichtswahrnehmung im Rahmen mathematischer Sprachförderung. Journal für Mathematik-Didaktik, 41, 191-236. https://doi.org/10.1007/s13138-020-00159-y.

Leiss, D., Blum, W., \& Messner, R. (2007). Die Förderung selbständigen Lernens im Mathematikunterricht - Problemfelder bei ko-konstruktiven Lösungsprozessen. Journal für Mathematik-Didaktik, 28(3/4), 224-248. https://doi.org/10.1007/BF03339347.

Leiss, D., Plath, J., \& Schwippert, K. (2019). Language and mathematics-key factors influencing the comprehension process in reality-based tasks. Mathematical Thinking and Learning, 21, 131-153. https://doi.org/10.1080/10986065.2019.1570835.

Leiss, D., Schukajlow, S., Blum, W., Messner, R., \& Pekrun, R. (2010). Zur Rolle des Situationsmodells beim mathematischen Modellieren - Aufgabenanalysen, Schülerkompetenzen und Lehrerinterventionen. Journal für Mathematik-Didaktik, 31(1), 119-141. https://doi.org/10.1007/s13138-010-0006-y.

Lingel, K., Neuenhaus, N., Artel, C., \& Schneider, W. (2014). Der Einfluss des metakognitiven Wissens auf die Entwicklung der Mathematikleistung am Beginn der Sekundarstufe I. Journal für MathematikDidaktik, 35, 49-77. https://doi.org/10.1007/s13138-013-0061-2.

Leopold, C., \& Leutner, D. (2015). Improving students' science text comprehension through metacognitive self-regulation when applying learning strategies. Metacognition and Learning, 10, 313-346. https:// doi.org/10.1007/s11409-014-9130-2.

Maaß, K., \& Engeln, K. (2018). Impact of professional development involving modelling on teachers and their teaching. ZDM Mathematics Education, 50, 273-285. https://doi.org/10.1007/s11858-01901048-6. 
Muthén, L. K., \& Muthén, B. O. (2018). Mplus user's guide: statistical analysis with latent variables. Bd. 7. Los Angeles: Muthén \& Muthén.

Paulus, C. (2009). Die „Bücheraufgabe“ zur Bestimmung des kulturellen Kapitals bei Grundschülern. Universität des Saarlandes. https://docplayer.org/18544402-Die-buecheraufgabe-zur-bestimmung.html. Zugegriffen: 22. Jan. 2019.

Perry, N.E., Hutchinson, L., \& Thauberger, C. (2008). Talking about teaching self-regulated learning: scaffolding student teachers' development and use of practices that promote self-regulated learning. International Journal of Educational Research, 47(2), 97-108. https://doi.org/10.1016/j.ijer.2007.11. 010.

Philipp, M., \& Schilcher, A. (2012). Selbstreguliertes Lesen. Ein Überblick über wirksame Leseförderansätze. Seelze: Klett.

Plath, J. (2019). Verstehensprozesse bei der Bearbeitung realitätsbezogener Mathematikaufgaben: Klassische Textaufgaben vs. Zeitungstexte. Journal für Mathematik-Didaktik. https://doi.org/10.1007/ s13138-019-00148-w.

Prediger, S., \& Krägeloh, N. (2015). Low achieving eighth graders learn to crack word problems: a design research project for aligning a strategic scaffolding tool to students' mental processes. ZDM Mathematics Education, 47, 947-962. https://doi.org/10.1007/s11858-015-0702-7.

Prediger, S., Wilhelm, N., Büchter, A., Gürsoy, E., \& Benholz, C. (2015). Sprachkompetenz und Mathematikleistung - Empirische Untersuchung sprachlich bedingter Hürden in den Zentralen Prüfungen 10. Journal für Mathematik-Didaktik, 36(1), 77-104. https://doi.org/10.1007/s13138-015-0074-0.

Reinhold, F., Reiss, K., Diedrich, J., Hofer, S., \& Heinze, A. (2019). Mathematische Kompetenz in PISA 2018 - aktueller Stand und Entwicklungen. In K. Reiss, M. Weis, E. Klieme \& O. Köller (Hrsg.), PISA 2018. Grundbildung in internationalen Vergleich (S. 187-209). Münster: Waxmann.

Reusser, K. (1989). Vom Text zur Situation zur Gleichung. Kognitive Simulation von Sprachverständnis und Mathematisierung beim Lösen von Textaufgaben. Habilitationsschrift. Bern: Universität Bern.

Richter, T., \& Schnotz, W. (2018). Textverstehen. In S. Buch, D. Rost \& J. Sparfeldt (Hrsg.), Handwörterbuch Pädagogische Psychologie (4. Aufl. S. 826-837). Weinheim: Beltz.

Schiepe-Tiska, A., Reiss, K., Obersteiner, A., Heine, J.H., Seidel, T., \& Prenzel, M. (2013). Mathematikunterricht in Deutschland: Befunde aus PISA 2012. In M. Prenzel, C. Sälzer, E. Klieme \& O. Köller (Hrsg.), PISA 2012: Fortschritte und Herausforderungen in Deutschland (S. 123-154). Münster: Waxmann.

Schlager, S. (2020). Zur Erforschung des Zusammenhangs zwischen Sprachkompetenz und Mathematikleistung. Oberflächlichkeit als potenzieller Moderator. Essener Beiträge zur Mathematikdidaktik. Wiesbaden: Springer Spektrum. https://doi.org/10.1007/978-3-658-31871-0.

Schlagmüller, M., \& Schneider, W. (2007). Würzburger Lesestrategie-Wissenstest für die Klassen 7-12 (WLST 7-12). Göttingen: Hogrefe.

Schmitz, A. (2019). Reading strategy instruction in $5^{\text {th }}$ grad: teachers' perspectives on promoting selfregulated reading in language and content area classrooms. Research in Subject Matter Teaching and Learning, 2, 16-31. https://doi.org/10.23770/rt1822.

Schmitz, A., Zeuch, N., Karstens, F., Meudt, S.-I., Jost, J., \& Souvignier, E. (2021). Leseförderung im Schul- und Unterrichtsalltag implementieren - Erste Erkenntnisse des Evaluationsprojekts BiSSEILe. In S. Gentrup, S. Henschel, K. Schotte, L. Beck \& P. Stanat (Hrsg.), Sprach- und Schriftsprachförderung wirksam gestalten: Evaluation umgesetzter Konzepte (S. 199-218). Stuttgart: Kohlhammer.

Schukajlow, S., \& Leiss, D. (2011). Selbstberichtete Strategienutzung und mathematische Modellierungskompetenz. Journal für Mathematik-Didaktik, 32(1), 53-77. https://doi.org/10.1007/s13138-0100023-X.

Schukajlow, S., Kaiser, G., \& Stillman, G. (2018). Empirical research on teaching and learning of mathematical modelling: a survey on the current state-of-the-art. ZDM Mathematics Education, 50, 5-18. https://doi.org/10.1007/s11858-018-0933-5.

Schünemann, N., Spörer, N., \& Brunstein, J.C. (2013). Integrating self-regulation in whole-class reciprocal teaching. A moderator-mediator analysis of incremental effects on fifth graders' reading comprehension. Contemporary Educational Psychology, 38, 289-305. https://doi.org/10.1016/j.cedpsych.2013. 06.002 .

Seidel, T. (2006). The role of student characteristics in studying micro teaching-learning environments. Learning Environments Research, 9, 253-271. https://doi.org/10.1007/s10984-006-9012-x.

Shanahan, C., Shanahan, T., \& Misischia, C. (2011). Analysis of expert readers in three disciplines: history, mathematics, and chemistry. Journal of Literacy Research, 43(4), 393-429. https://doi.org/10.1177/ $1086296 \times 11424071$. 
Souvignier, E., \& Mokhlesgerami, J. (2006). Using self-regulation as a framework for implementing strategy instruction to foster reading comprehension. Learning and Instruction, 16, 57-71. https://doi. org/10.1016/j.learninstruc.2005.12.006.

Souvignier, E., Trenk-Hinterberger, I., Adam-Schwebe, S., \& Gold, A. (2008). Frankfurter Leseverständnistest für 5. und 6. Klassen (FLVT 5-6). Göttingen: Hogrefe.

Van Dijk, T., \& Kintsch, W. (1983). Strategies of discourse comprehension. New York: Academic Press.

Verschaffel, L., Greer, B., \& de Corte, E. (2000). Making sense of word problems. Lisse: Swets.

Vieluf, S., Kaplan, D., Klieme, E., \& Bayer, S. (2012). Teaching practices and pedagogical innovation: evidence from TALIS. Paris: OECD Publishing.

Wagner, W., Helmke, A., \& Rösner, E. (2009). Deutsch Englisch Schülerleistungen International. Dokumentation der Erhebungsinstrumente für Schülerinnen und Schüler, Eltern und Lehrkräfte. Materialien zur Bildungsforschung, Bd. 25/1. Frankfurt a. M.: DIPF.

Weinstein, C.E., \& Mayer, R. E. (1986). The teaching of learning strategies. In M. Wittrock (Hrsg.), Handbook of research on teaching (Bd. 3, S. 315-327). New York, London: Macmillan.

Weis, M., Doroganova, A., Hahnel, C., Becker-Mrotzek, M., Lindauer, T., Artelt, C., \& Reiss, K. (2019). Lesekompetenz in PISA 2018 - Ergebnisse in einer digitalen Welt. In K. Reiss, M. Weis, E. Klieme \& O. Köller (Hrsg.), PISA 2018. Grundbildung in internationalen Vergleich (S. 47-80). Münster: Waxmann.

Weiß, R. H. (2006). Grundintelligenztest Skala 2 - Revision. CFT 20-R mit WS/ZF-R. Göttingen: Hogrefe.

Whitebread, D. (2000). Interpretations of independent learning in the early years. International Journal of Early Years Education, 8(3), 243-252. https://doi.org/10.1080/09669760050156785.

Wimmer, H., \& Mayringer, H. (2014). Salzburger Lese-Screening für die Schulstufen 2-9. Göttingen: Hogrefe.

Zimmerman, B.J. (2000). Attaining self-regulation: a social cognitive perspective. In M. Boekaerts, P. R. Pintrich \& M. Zeidner (Hrsg.), Handbook of self-regulation (S. 13-39). San Diego: Academic Press. https://doi.org/10.1016/B978-012109890-2/50031-7. 